\title{
Article
}

\section{Innovative Biotechnology for Generation of Cardiac Tissue}

\author{
Greta Ionela Barbulescu 1,2,*(D), Florina Maria Bojin 1,3,*, Valentin Laurentiu Ordodi ${ }^{3,4}$, Iacob Daniel Goje 5 (D), \\ Taddeus Paul Buica ${ }^{3}$, Oana Isabella Gavriliuc ${ }^{1,3}$, Flavia Baderca ${ }^{6}$, Teodora Hoinoiu ${ }^{2,7}$ and Virgil Paunescu ${ }^{1,3}$
}

1 Immuno-Physiology and Biotechnologies Center, Department of Functional Sciences, "Victor Babes" University of Medicine and Pharmacy, No. 2 Eftimie Murgu Square, 300041 Timisoara, Romania; gavriliuc.oana@umft.ro (O.I.G.); vpaunescu@umft.ro (V.P.)

2 Department of Clinical Practical Skills, "Victor Babes" University of Medicine and Pharmacy, No. 2 Eftimie Murgu Square, 300041 Timisoara, Romania; tstoichitoiu@umft.ro

3 Clinical Emergency County Hospital "Pius Brinzeu" Timisoara, Center for Gene and Cellular Therapies in the Treatment of Cancer Timisoara-OncoGen, No. 156 Liviu Rebreanu, 300723 Timisoara, Romania; valentin.ordodi@upt.ro (V.L.O.); taddeus.b90@gmail.com (T.P.B.)

4 Faculty of Industrial Chemistry and Environmental Engineering, "Politehnica" University Timisoara, No. 2 Victoriei Square, 300006 Timisoara, Romania

5 Department of Internal Medicine I, Medical Semiology I, “Victor Babes” University of Medicine and Pharmacy, No. 2 Eftimie Murgu Square, 300041 Timisoara, Romania; daniel.goje@umft.ro

6 Department of Microscopic Morphology, Histology, "Victor Babes" University of Medicine and Pharmacy, No. 2 Eftimie Murgu Square, 300041 Timisoara, Romania; baderca.flavia@umft.ro

7 Clinic of Burns, Plastic and Reconstructive Surgery, "Pius Brinzeu" Clinical Emergency County Hospital, No. 156 Liviu Rebreanu, 300723 Timisoara, Romania

check for updates

Citation: Barbulescu, G.I.; Bojin, F.M.; Ordodi, V.L.; Goje, I.D.; Buica, T.P.; Gavriliuc, O.I.; Baderca, F.; Hoinoiu, T.; Paunescu, V. Innovative Biotechnology for Generation of Cardiac Tissue. Appl. Sci. 2021, 11, 5603. https://doi.org/10.3390/ app11125603

\section{Academic Editors:}

Szerb Elisabeta Ildyko, Cseh Liliana, Manea Florica and Tudose Ramona

\section{Received: 28 April 2021}

Accepted: 14 June 2021

Published: 17 June 2021

Publisher's Note: MDPI stays neutral with regard to jurisdictional claims in published maps and institutional affiliations.

Copyright: (c) 2021 by the authors. Licensee MDPI, Basel, Switzerland. This article is an open access article distributed under the terms and conditions of the Creative Commons Attribution (CC BY) license (https:// creativecommons.org/licenses/by/ $4.0 /)$.
* Correspondence: barbulescu.greta@umft.ro (G.-I.B.); florinabojin@umft.ro (F.M.B.); Tel.: +40-733177583 (G.-I.B.)

Abstract: Heart transplantation remains the only curative treatment for end-stage heart failure. This life-saving option continues to be limited by the low number of organ donors, graft rejection and adverse effects of immunosuppressants. Engineering bioartificial hearts from acellular native-derived scaffolds and stem cells has gained attention because of its potential to overcome these limitations. In this study, rat hearts $(n=20)$ were decellularized by means of coronary perfusion with $1 \%$ sodium dodecyl sulfate (SDS) in a modified Langendorff device. The electrical field behavior of the SDS molecule was studied and it was assumed that when applying an alternating current, the exposure time of the tissue to the detergent might decrease. To repopulate the decellularized extracellular matrix (ECM), human mesenchymal stem cells (hMSCs) were used, induced to differentiate into cardiomyocytes (CMs) with 5-azacytidine (5-aza). The results showed no cellular debris and an intact ECM following decellularization. Decellularization in the presence of an electric field proved to be faster, decreasing the potential risk of ECM damage due to the detergent. After cell seeding and culturing of eight scaffolds with hMSCs, the recellularization process was analyzed using optic microscopy (OM), which showed cells suggestive for CMs. This study presents a novel and efficient decellularization protocol using an electric field and suggests that hMSCs can be useful in the generation of a bioartificial heart.

Keywords: cardiac decellularization; decellularized extracellular matrix; mesenchymal stem cells; regenerative medicine; recellularization; engineering bioartificial heart

\section{Introduction}

Cardiovascular diseases (CVDs) are the leading cause of global mortality, and the total number of patients with heart failure still continues to rise [1]. More than 64.3 million people are living with heart failure worldwide [2]. Despite substantial progress in recent years, heart function is fully restored only after cardiac transplantation. The main challenges associated with this are the poor availability of organ donors, as well as the 
long-term immunosuppressants needed after surgery [3]. The idea of creating a bioartificial heart could at least theoretically solve the problems seen in allogenic human heart transplantation surgery.

Three-dimensional (3D) cardiac tissue generation requires specialized cardiac cells and a 3D heart scaffold on which the seeded cells can form a mature tissue capable of sustaining complex cardiac functions [4,5]. Synthetic heart scaffolds have been used but showed a lack of characteristics required for cell attachment, with no dynamic reciprocity between them [6,7]. The decellularized extracellular matrix (ECM) remains the perfect scaffold because it preserves the complexity of the native organ. It maintains hierarchical vasculature, acts as the reservoir of growth factors and enhances their actions, and it provides structural support for cells to reside, proliferate and differentiate $[8,9]$. The first successful bioartificial rodent heart was created by Ott et al. in 2008. Researchers described obtaining a native-derived acellular 3D ECM through coronary perfusion with three different detergent solutions. The rat heart scaffold was repopulated with neonatal rat cardiomyocytes (CMs) and rat endothelial cells (ECs) [10].

The decellularization process involves washing out all the cells and cellular remnants such as deoxyribonucleic acid (DNA), membranes and cytosolic materials [11,12]. Different decellularization methods have been used over time, including physical, chemical and enzymatic methods and combinations of them [13,14]. Ott et al. showed that the use of sodium dodecyl sulfate (SDS) gave better results compared with other detergents such as Triton-X100, polyethylene glycol (PEG) or enzyme-based protocols [10]. Weymann et al. decellularized a porcine heart using $4 \%$ SDS for $12 \mathrm{~h}$ with a DNA concentration that was significantly decreased after antegrade perfusion in a Langendorff device [15,16]. In 2018, Park et al. studied the effect of pulsatile perfusion for the decellularization of a rat heart, demonstrating a more profound and efficient decellularization in the pulsatile group [17].

Cells are needed for the recellularization of the decellularized ECM in order to engineer a bioartificial heart. Such a complex organ as the heart needs an important number of cells specialized to differentiate into ECs, CMs, smooth muscle cells (SMCs), fibroblasts and conducting cells [18]. The use of stem cells to create a bioartificial heart has gained a lot of support in recent years. Embryonic stem cells [19], human mesenchymal stem cells (hMSCs) [20] and induced pluripotent stem cells (iPSCs) [21] were studied and differentiated into cardiomyocytes in several studies with promising results. Mesenchymal stem cells (MSCs) are multipotent stem cells found in bone marrow, which are capable of differentiating into cardiac cells. They have been used in the treatment of heart disease [22], showing a lot of advantages, such as a strong capacity for proliferation, ease of availability and low immunogenicity [23-25].

The purpose of this study was to obtain a bioartificial rat heart from a decellularized heart used as a matrix for grafting hMSCs. The decellularization protocol in the presence of an electric field decreases the exposure time of the tissue to the detergent and preserves the ECM components. Recellularization of the 3D ECM at a later timepoint was performed using hMSCs differentiated into cardiomyocytes with 5-azacytidine (5-aza).

\section{Materials and Methods}

\subsection{Animals}

Twenty adult male Sprague-Dawley rats of 250-350 g (12-16 weeks old) were used for the generation of whole decellularized heart ECM. All rats were anesthetized with sevoflurane (AbbVie Deutschland GmbH \& Co. KG, Wiesbaden, Germany) induction at $8 \%$ and maintenance at $3.5 \%$, delivered in oxygen by means of a nose cone. A median laparotomy was performed to gain access to the inferior vena cava and perform systemic heparinization using unfractionated heparin (Pan Pharma GmbH, La Selle-en-Luitré, France) at $3.0 \mathrm{IU} / \mathrm{g}$ of body weight. The thorax was opened via a median sternotomy and the heart was removed from the chest, making sure that the ascending aorta had a suitable length for subsequent cannulation. Before the heart explantation, the cava and pulmonary veins, the pulmonary artery and the thoracic aorta were transected. The heart was washed with a 
cold isotonic saline solution containing anticoagulant to remove eventual blood from the coronary system and heart cavities. It was attached to the Langendorff device by fixing the aorta on the 18-gauge needle using a 3-0 non-absorbable surgical suture (Figure 1a-d).

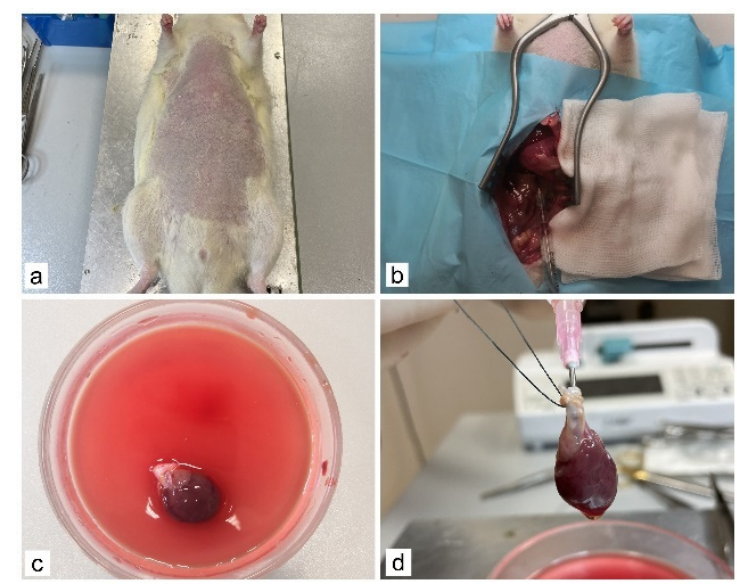

Figure 1. Heart explantation. $(\mathbf{a}, \mathbf{b})$ Removal of the heart from the chest. (c) Preparation of the heart to be washed with isotonic saline solution containing anticoagulant. (d) Attachment of a needle into the ascending aorta to allow for retrograde coronary perfusion.

\subsection{Whole-Heart Decellularization through Coronary Perfusion}

The rat hearts $(n=20)$ were decellularized using a modified Langendorff experimental device, previously presented in our studies. The experimental device respects the principle described by Langendorff. The decellularization solution is aspirated from the decellularization chamber by the peristaltic pump and reintroduced into the heart via the cannula. The perfusion pressure of the heart is the pressure on the discharge branch of the peristaltic pump and it is monitored by the pressure transducer. The operation of the peristaltic pump is controlled by the automation system. The output of this system reaches a power amplifier and via a solid-state relay it controls the peristaltic pump function [26,27].

Half of the hearts $(n=10)$ were decellularized in the absence of the electric field (control group) and the other half $(n=10)$ in the presence of an alternating rectangular electric field. The hearts were perfused with $1 \%$ sodium dodecyl sulfate (SDS; Thermo Scientific, Waltham, MA, USA) in deionized water for $16 \mathrm{~h}$ at about $80 \mathrm{mmHg}$. A peristaltic pump was used for the perfusion of the heart, recirculating the decellularization solution during the entire course of the experiment. One advantage of this system is a relatively decreased volume of decellularization solution used, which is approximately $150 \mathrm{~mL}$. The heart was totally covered with solution within the glass container, constituting the electrochemical cell. Two stainless steel plate-type electrodes (the surface of each electrode was approximately $14 \mathrm{~cm}^{2}$ ) were placed on the container to generate the alternative electric field required for decellularization. The two electrodes were reachable from the exterior of the electrochemical cell. For generation of alternative electric field, a generator of functions was used, programmed to produce a rectangular electric signal, with a constant frequency $(20 \mathrm{hHz})$ and amplitude $(100 \mathrm{~mA})$, corresponding to $7.14 \mathrm{~mA} / \mathrm{cm}^{2}$ current density. Due to the electrical field behavior of the SDS molecule, it was assumed that the detergent would more efficiently penetrate the cellular membranes, allowing for a faster decellularization. During all the decellularization experiment procedures, $10-\mu \mathrm{L}$ samples were taken from the decellularization solution every $30 \mathrm{~min}$ for spectrophotometric measurement of the DNA and total protein concentrations. The hearts were weighed before the decellularization process, with no significant differences between the two groups. For the decellularization process, the aortic cannula was inserted into the ascending aorta and secured with a surgical suture to allow for retrograde coronary perfusion with a flow of $8 \mathrm{~mL} / \mathrm{min}$ (Figure $2 \mathrm{a}-\mathrm{g}$ ). 

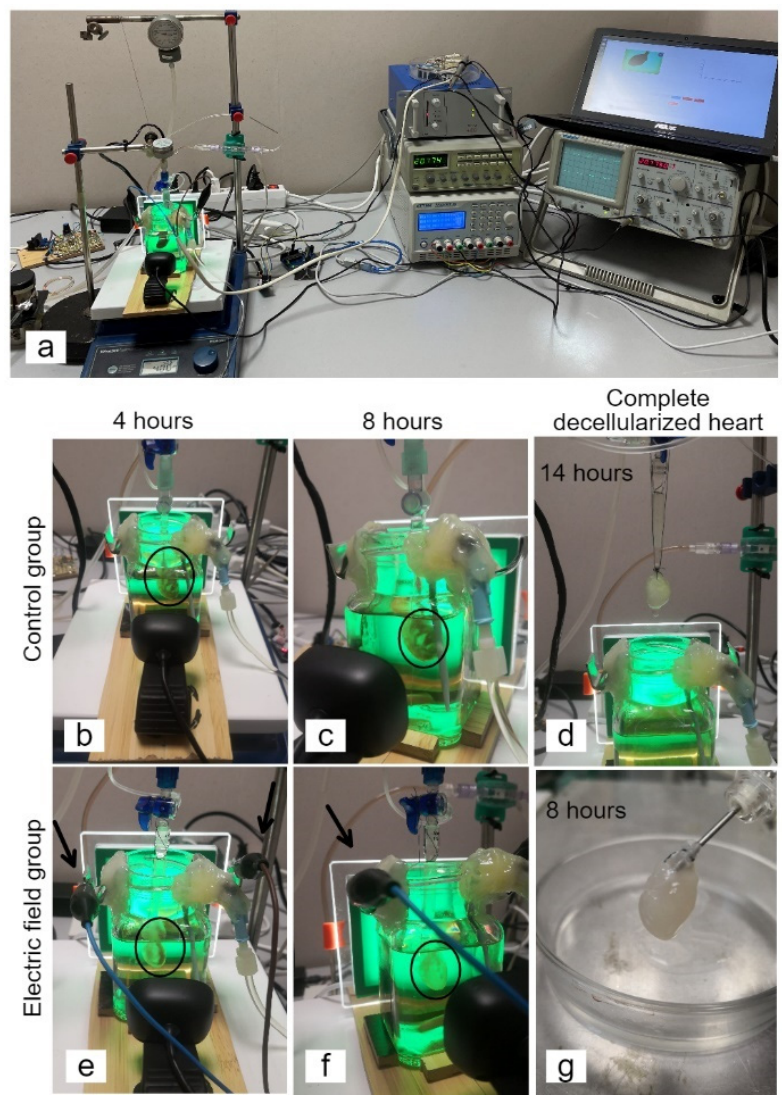

Figure 2. System set-up of the modified Langendorff experimental device. (a) General view of the setup. (b) 4-h and (c) 8-h retrograde perfusion of the rat heart with 1\% SDS in the control group (modified Langendorff experimental device in the absence of an electric field). (d) A complete decellularized rat heart from the control group after $14 \mathrm{~h}$ of perfusion with $1 \%$ SDS. (e) $4-\mathrm{h}$ and (f) 8-h retrograde perfusion of the rat heart with $1 \%$ SDS in the presence of an alternating rectangular electric field. The electric contacts with the power supply are marked by black arrows. (g) A complete decellularized rat heart from the electric field group after $8 \mathrm{~h}$ of perfusion with $1 \%$ SDS (the heart becomes translucent when all the cellular material is washed out, while preserving the anatomy, vascular network and ECM characteristics of the native organ).

It is to be noted that after its use, the detergent should be removed from the remaining extracellular matrix (ECM), washing it with phosphate buffered saline (PBS; Sigma-Aldrich, St. Louis, MO, USA) and deionized water. The hearts were perfused and stored with 10,000 IU/mL penicillin-streptomycin (Pen/Strep 10,000 IU/mL; Sigma-Aldrich, St. Louis, $\mathrm{MO}, \mathrm{USA}$ ) in PBS before recellularization.

\subsection{Extracellular Matrix Characterization}

After decellularization, several areas from the heart, evenly spaced between the base and the apex ( $n=6-8$ tissue samples per heart), were isolated, fixed in $4 \%$ buffered formaldehyde solution (Roti-Histofix, Carl Roth $\mathrm{GmbH}$, Karlsruhe, Germany), embedded in paraffin and cut with a microtome into histological sections of $5 \mu \mathrm{m}$. Before staining, the specimens were deparaffinized with xylene (Sigma-Aldrich, St. Louis, MO, USA; Merck \& Co., Kenilworth, NJ, USA), treated with graded ethanol solutions and rehydrated according to the manufacturer's protocols. The hematoxylin and eosin (H\&E) staining method (Sigma-Aldrich, St. Louis, MO, USA; Merck \& Co., Kenilworth, NJ, USA) was first used for characterization of the ECM. The staining was performed by incubating the tissue in hematoxylin solution for $5 \mathrm{~min}$, followed by immersion in eosin for $30 \mathrm{~s}$, and the washing steps were performed with tap water. H\&E staining was used for the visualization of residual cellular remnants (basophilic nuclei appear in blue/black and eosinophilic cytoplasm 
and collagen in pink). For a better characterization of the ECM, sections were also stained via Masson's trichrome staining protocol (Bio-Optica, Milano, Italy) for visualization of collagenous connective tissue fibers. In Masson's trichrome staining, hematoxylin (5 min incubation) stains the nuclei of the cells black, acid fuchsin ( 3 min immersion) dyes the cytoplasm red, and the connective tissue is stained with aniline blue (10 min). Argentic impregnation (Sigma-Aldrich, St. Louis, MO, USA; Merck \& Co., Kenilworth, NJ, USA) lends itself especially well to the demonstration of reticular connective tissue. The reticulum is sharply impregnated, appearing brownish-black. If the processes of oxidation and reduction are accomplished, the background is almost colorless, with elements other than fibers (nuclei or cytoplasm) being silver-negative. Characterization of ECM content was also conducted, utilizing the orcein staining technique, according to the manufacturer's protocol (Orcein for elastic fibers; Bio-Optica, Milano, Italy) for the visualization of elastic fibers, which appeared in dark brown. All imaging was performed under a light microscope Leica DM750 Clinical Microscope (Leica Microsystems, Wetzlar, Germany) and a Panthera L Microscope (Motic, Richmond, CA, USA). Before the characterization of the extracellular matrix, the hearts from the control group and the electric field group were kept for $16 \mathrm{~h}$ in the decellularization solution. The process was considered finished when there were no traces of myocardial tissue remaining from the macroscopic point of view.

Scanning electron microscopy (SEM) was used to characterize the morphological changes following decellularization. The protocol involved 2.5\% $(v / v)$ buffered glutaraldehyde (in PBS) fixation for $1 \mathrm{~h}$ at room temperature, followed by washing with PBS several times (three times, 15 min each), 1\% osmium tetroxide $\left(\mathrm{OsO}_{4}\right.$; Sigma-Aldrich, St. Louis, MO, USA; Merck \& Co., Kenilworth, NJ, USA) post-fixation for $1 \mathrm{~h}$, followed again by fresh buffer. After osmium tetroxide fixation, the specimens were dehydrated using ethanol solutions of increasing concentrations (30\%, 50\%, 70\%, 90\% and 100\%), hexamethyldisilazane (HMDS; Sigma-Aldrich, St. Louis, MO, USA; Merck \& Co., Kenilworth, NJ, USA) and then air-dried. The dried tissue blocks were visualized with a FEI Quanta 3D FEG electron microscope (FEI Company, Eindhoven, NL, USA). The decellularization was quantified on 4-6 individual SEM fields/experiment.

Another method used for the characterization of the ECM involved determining the concentration of deoxyribonucleic acid (DNA) and proteins in the decellularization medium using a NanoDrop 1000 Spectrophotometer (Thermo Scientific, Waltham, MA, USA). The samples (10 $\mu \mathrm{L}$ each) were collected using an automatic micropipette directly from the decellularization tank at a preset protocol time interval (30 min). DNA and protein concentrations were expected to increase and reach a plateau when the decellularization was completed. The decellularization protocols using the modified Langendorff experimental device (the control group versus the group using the alternating rectangular electric field) could be compared according to the time needed for a complete decellularization. It is therefore desirable to make the decellularization process quicker so that the exposure time of the heart to the detergent is reduced because of its potential harmful action on the ECM.

\subsection{Cell Culture}

In order to generate a bioartificial rat heart, human mesenchymal stem cells (hMSCs) isolated from adult human bone marrow (Lonza Group, Basel, Switzerland) were used. They were expanded and cultivated in alpha-minimum essential medium (MEM; Invitrogen, CA, USA) supplemented with 10\% fetal bovine serum (FBS; Promo Cell, Heidelberg, Germany) and 1\% Pen/Strep 10,000 IU/mL solution. The cells were cultured at $37{ }^{\circ} \mathrm{C}$ in a $5 \% \mathrm{CO}_{2}$ atmosphere. The medium was replaced every $72 \mathrm{~h}$. The cells were removed from the culture plate using $0.25 \%$ Trypsin-EDTA (Sigma-Aldrich, St. Louis, MO, USA) when reaching $80-90 \%$ confluence, then centrifugated (10 $\mathrm{min}, 300 \mathrm{~g}$ ) and recultivated in T75 culture flasks at a density of 10,000 cells $/ \mathrm{cm}^{2}$ for an optimal proliferation. For every cell seeding experiment $(n=8)$ the same number of hMSCs were delivered $\left(\sim 5-7 \times 10^{9}\right.$ cells). Most of the previous studies on the recellularization of the whole decellularized heart 
extracellular matrix used approximately the same number of cells, including the pioneering work of Ott et al. from 2008 [10].

\subsection{Recellularization of Whole Rat Heart}

With the aim of determining the optimal strategy for recellularization, two different delivery methods were examined. For the reconstruction of the whole heart, extracellular matrix $(\mathrm{ECM})(n=8)$ obtained through decellularization from the control group $(n=4)$ and the electric field group $(n=4)$ was used. The decellularized hearts were initially perfused for $72 \mathrm{~h}$ with PBS. The ascending aorta was cannulated with sterile 18-gauge cannula (Radnoti Glass) and the heart was mounted in a bioreactor and perfused with $37^{\circ} \mathrm{C}$ oxygenated cell medium for $24 \mathrm{~h}$ at a coronary rate flow of $8 \mathrm{~mL} / \mathrm{min}$ in order to create the optimal conditions for the ECM seeding bed. In this phase, the nonspecific DNA methylation inhibitor 5-azacytidine (5-aza) was used as an agent to differentiate hMSCs into cardiomyocytes (CMs). Treatment with 5-aza (10 $\mu \mathrm{M}$, Sigma-Aldrich, St. Louis, MO, USA) has been shown to stimulate in vitro cardio myogenesis in hMSCs $[28,29]$. The first protocol required the hMSCs $\left(\sim 5-7 \times 10^{9}\right.$ cells $)$ to stay for $24 \mathrm{~h}$ on plates with 5-aza and, after trypsinization, to be resuspended in MSC culture media (MEM supplemented with $10 \%$ FBS and $1 \%$ Pen/Strep 10,000 IU / mL). For the cell seeding, about half of these cells were delivered by infusion through the patent aorta for $24 \mathrm{~h}$ and half were delivered through 4 injections of $200 \mu \mathrm{L}$, each in the thickness of the ECM with a 27-G needle and a 1-cc tuberculin syringe. The second protocol delivered the hMSCs directly with 5-aza suspended in the culture media via infusion through the aorta for $24 \mathrm{~h}$ and through 4 injections. In both protocols, the MSC culture media was changed every $24 \mathrm{~h}$. The hearts were kept for 3 weeks in a tissue-culture incubator in a $5 \% \mathrm{CO}_{2}$ atmosphere with additional humidified carbogen $\left(5 \% \mathrm{CO}_{2}\right.$ and $\left.95 \% \mathrm{O}_{2}\right)$ injected into the medium reservoir (Figure $3 a, b)$. At the end of the experiments, the H\&E staining protocol was used for the characterization of the recellularized ECM as described.

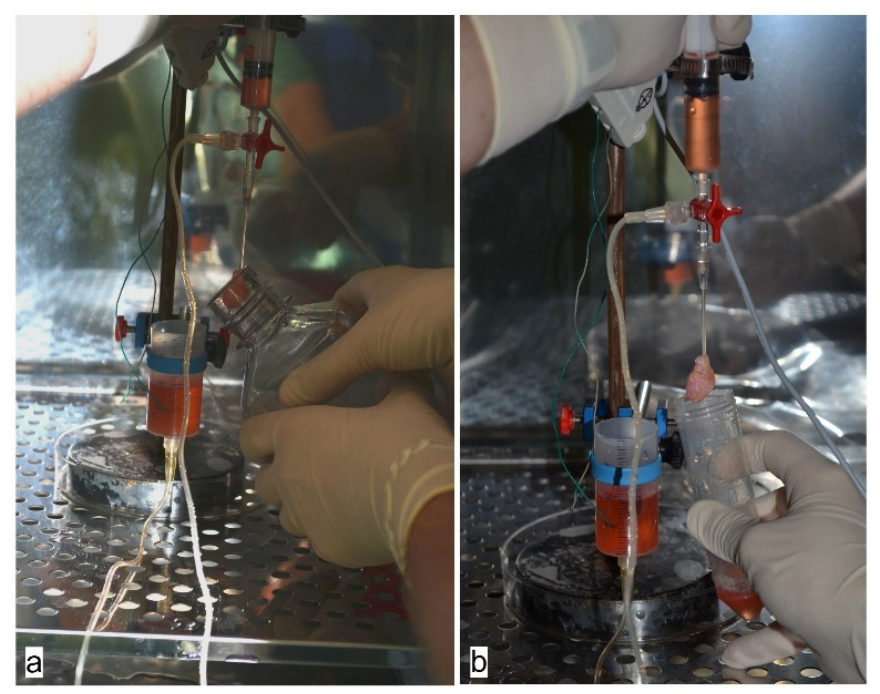

Figure 3. Cardiac tissue engineering using a perfusion bioreactor system. $(\mathbf{a}, \mathbf{b})$ The heart is kept in a tissue-culture incubator by cannulating the ascending aorta.

\section{Results}

\subsection{Generation of an Acellular Rat Heart using Perfusion Decellularization}

Decellularization was considered finished when there was no trace of myocardial tissue remaining. Macroscopic analysis of the ECM after the decellularization process showed an intact 3D geometry and the maintenance of the atrial, ventricular and valvular architecture (Figure 2d,g). Photographs taken during decellularization showed a more efficient and rapid decellularization in the electric field group compared with the control group (Figure $2 \mathrm{~b}-\mathrm{g}$ ). 
The coronary vessel tree's integrity after decellularization was verified by injecting trypan blue solution through the connected cannula. The entire process was recorded and the entry of the blue solution into the coronary system was able to be visualized, without any signs of problems with the caliber of the vessels (Figure $4 a-h)$.

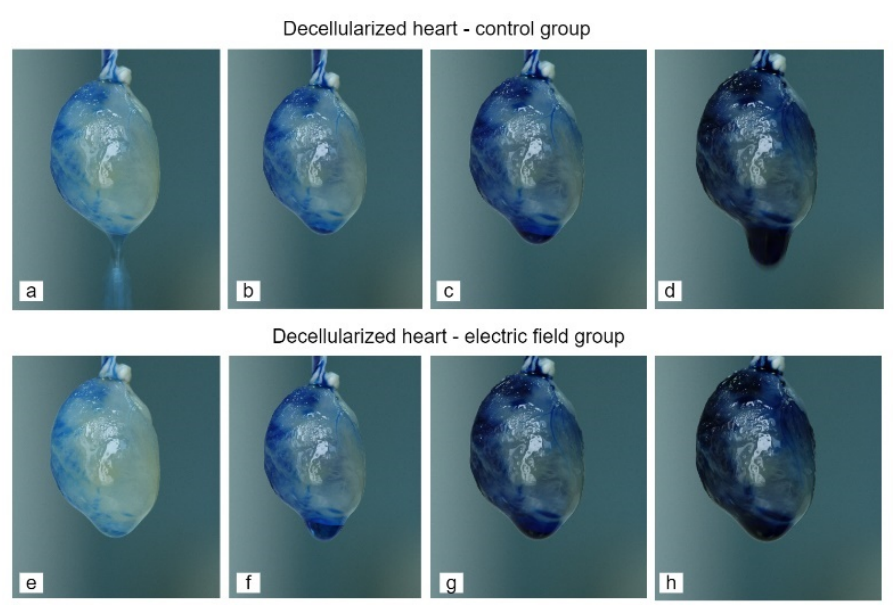

Figure 4. Coronary vessel tree integrity. Perfusion of decellularized ECM with trypan blue solution to visualize the intact coronary vasculature. (a-d) Control group of decellularized hearts (30 s); (e-h) Electric field group of decellularized hearts (30 s).

\subsection{Evaluation of Matrix Content}

The efficiency of decellularization was validated through a number of different methods. As an initial step, the simple H\&E staining method demonstrated the removal of cells, while preserving the 3D ECM. The preservation of collagen fibers and clearance of nuclear content from the decellularized scaffolds was highlighted using Masson's trichrome staining. Orcein staining and argentic impregnation visualized the elastic fibers and the reticulin fibers, respectively. The scanning electron microscopy (SEM) of the 3D cardiac framework strengthened the results of the optical microscopy (OM), showing reserved collagen fibers with no cellular components remaining. The evaluation of the matrix content using microscopy showed no significant differences between the scaffolds generated by the two decellularization protocols (Figure $5 \mathrm{a}-\mathrm{j}$ ).

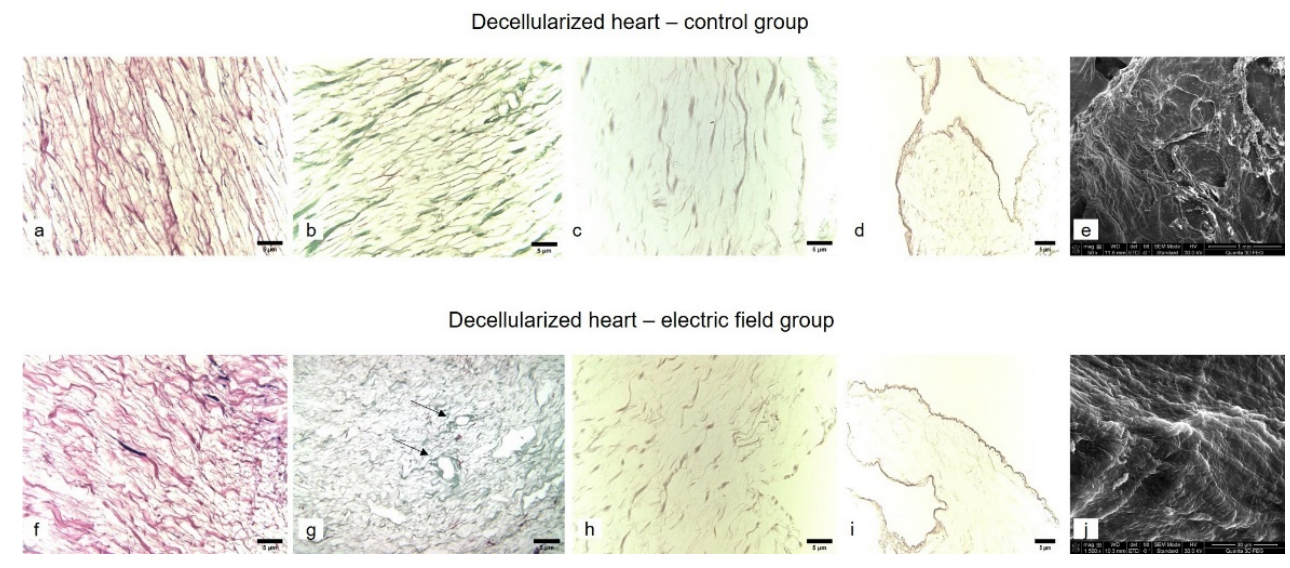

Figure 5. Extracellular matrix evaluation. (a,f) H\&E staining of thin sections from the rat hearts perfused with $1 \%$ SDS $(\mathrm{Ob} 40 \times)$ showed a uniform ECM, with no intact cells, nuclei or basophilic structures. (b,g) Decellularized myocardial ECM scaffold stained with Masson's trichrome staining protocol $(\mathrm{Ob} 40 \times)$ showed the absence of black nuclei; however, collagen was still present, indicated by the blue staining; in (g) the arrows indicate two blood vessels, identified by the thickness of the wall. (c,h) Argentic impregnation $(\mathrm{Ob} 40 \times)$ visualized preserved reticulin fibers. (d,i) Orcein staining $(\mathrm{Ob} 40 \times)$ of the ECM showed parallel elastic fibers. (e,j) SEM of the ECM showed that cells throughout tissue layers were removed, preserving the collagen fiber organization and structure. 
Comparing decellularization in the control group with decellularization in the electric field group regarding DNA and protein concentrations, the latter proved to be time-saving, taking about $8 \mathrm{~h}$ for the process to be completed. In the control group, the amount of time needed for the process was about $14 \mathrm{~h}$. The final moment of decellularization could be settled, considered as the time when the concentration of the two determined analytes (DNA and proteins) became constant (Figure $6 \mathrm{a}, \mathrm{b}$ ). The results provide evidence that alternative electric field decellularization is more efficient, and the matrix integrity is completely preserved. The total number of decellularized hearts $(n=20)$ was statistically significant, providing reliable results.

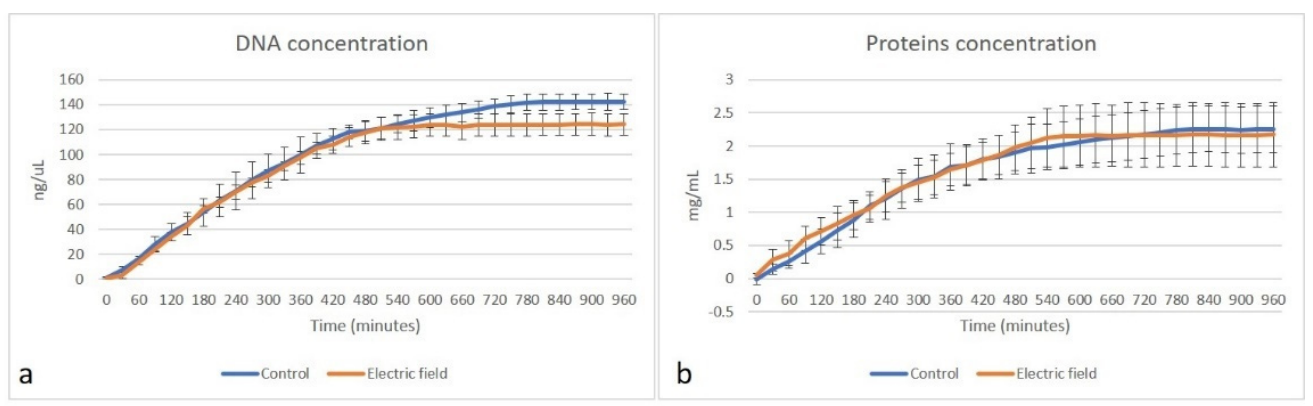

Figure 6. Total DNA and protein concentrations measured in decellularization solution. Comparison of DNA and protein concentrations in washing fluid during the development of heart decellularization using the modified Langendorff experimental device revealed a quasilinear increase in the parameters and a shorter average decellularization time needed for the process when using an electrical current. (a) The DNA concentrations for the control group reached the plateau phase at $840 \mathrm{~min}$, whereas for the electric field group the plateau phase was reached at 480-540 $\mathrm{min}$; (b) protein concentrations for the control group reached the plateau phase at $840 \mathrm{~min}$, and for the electric field group the protein plateau phase was reached at 480-540 $\mathrm{min}$.

\subsection{Formation of Bioartificial Whole Rat Heart}

The bioartificial rat hearts $(n=8)$ were macroscopically (Figure 7a-d) and microscopically characterized (Figure 8a-d). The two recellularization protocols involved a combined approach to recellularizing the whole heart, through the direct injection of hMSCs into the rat heart, in addition to infusion through the patent aorta for $24 \mathrm{~h}$. From the macroscopic point of view, well-defined cardiac walls, both atria and ventricles were observed. The blood vessels were visible through the thickness of the heart. Fibrous bands, which could be the fibrous cytoskeleton of the heart, were concentrated at the base of the ventricular mass. $H \& E$ staining from different regions of the recellularized hearts confirmed the presence of an important number of cells distributed throughout the acellular scaffold. Histologically, a normal myocardium contains cardiomyocytes that are densely packed and form a syncytium mass. Here, the examined tissue contained loosely arranged cardiomyocyte-like cells that had visible ramifications that connected, evident cross-striations, and detectable nuclei. Although the histological architecture was not normal, a structural organization was still discernable. On the examined material, approximately $20 \%$ of the matrix contained cells with evident nuclei, with alternating acellular areas and zones of dense cellularity. The optical microscopy $(\mathrm{OM})$ did not show any significant differences between the hearts recellularized with 5-aza using the two protocols (Figure 8a-d). 

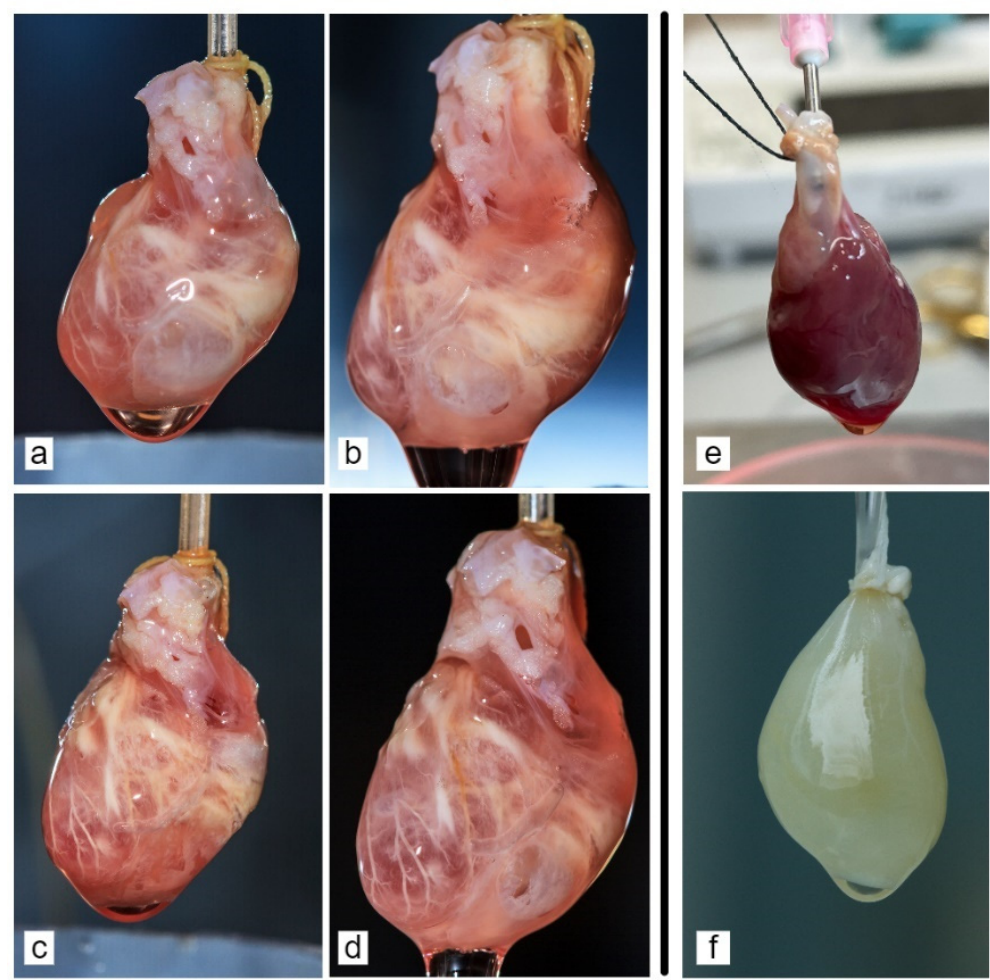

Figure 7. (a-d) The macroscopic view of the recellularized scaffold using hMSCs. (e) Freshly isolated native rat heart. (f) Decellularized rat heart using 1\% SDS in the modified Langendorff experimental device.

First protocol of ECM scaffold recellularization with hMSCs

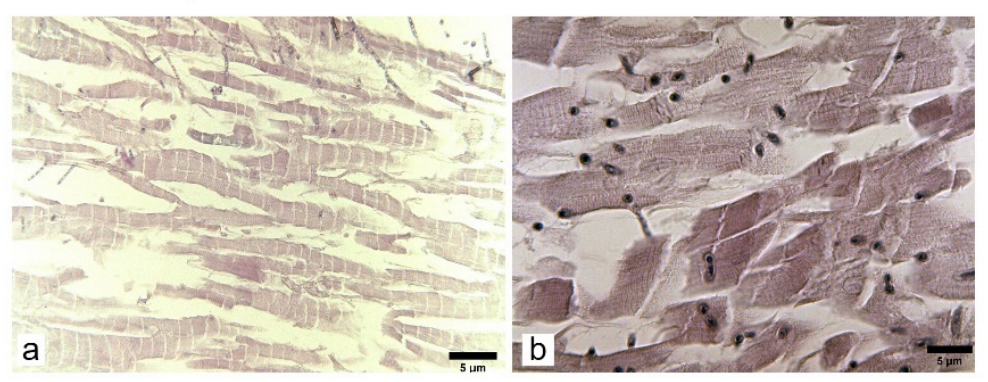

Second protocol of ECM scaffold recellularization with hMSCs

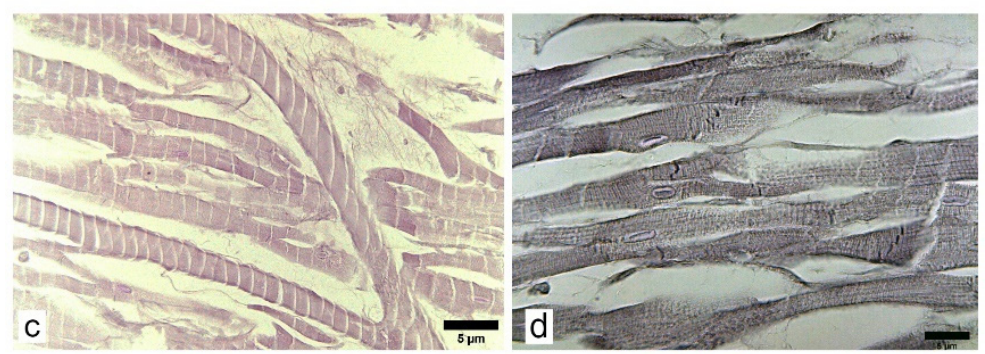

Figure 8. Histological analysis of recellularized rat heart scaffolds. H\&E staining showed cells that resembled cardiomyocytes, arranged in fibers exhibiting cross-striations and eosinophilic cytoplasm, which centrally presented the formation of a nucleus. (a-Ob $40 \times ; \mathbf{b}-\mathrm{Ob} 100 \times)$ First protocol of ECM scaffold recellularization with hMSCs. (c-Ob 40×; d-Ob 100×) Second protocol of ECM scaffold recellularization with hMSCs. 
Further experiments need to be conducted for complete cellular coverage in the recellularized rat heart as functional cardiac tissue requires viable cells for contractility and gap junction formation.

\section{Discussion}

The idea of building a bioartificial heart is not new. There has been a considerable amount of research in this field and yet many problems have remained unsolved. However, the current results are promising because the concept of whole-heart engineering is rapidly evolving $[18,30]$. Heart tissue engineering requires a scaffold so the stem cells can rebuild the muscle and vascular structures. The role of the ECM has been proven to be more significant than previously thought [31]. Previous studies have shown that only the whole-organ decellularization process creates a 3D ECM that mimics nature's design perfectly [10]. This design cannot be reproduced by any synthetic material, even though important attempts were made in this field [32,33].

This study presents an optimal decellularization protocol using sodium dodecyl sulfate (SDS) as a decellularization agent. This agent was chosen because important studies have shown its superiority when compared with other detergents such as Triton-X-100, PEG and trypsin [10,34]. Decellularization was performed using a modified Langendorff experimental device created in our lab, as shown in previous works $[26,27]$. This prototype provided efficient decellularization, taking about $14 \mathrm{~h}$ for the control rat hearts to become fully decellularized. Furthermore, we have demonstrated an alternative electric field system, apparatus and method of use for the preparation of decellularized rat hearts. The results supplied evidence that alternative electric decellularization was more efficient and time-saving, requiring about $8 \mathrm{~h}$ for the process to be completed. Every experiment was photographed for $16 \mathrm{~h}$. As soon as the heart became completely translucent, the DNA and the total protein concentrations from the decellularization solution started to reach a plateau. The efficiency of the decellularization process was proven using optical and electronic microscopy. Thus, the generation of a decellularized rat heart with no residual nuclear material was possible. Another important component to check when performing a decellularization protocol is the integrity of the coronary vessel tree [21]. It was proved that the coronary vessel tree within the decellularized ECM was preserved after decellularization by injecting trypan blue solution through the connected cannula.

The decellularized ECM present remains the best scaffold for tissue engineering but it has to deal with the difficult task of the complete removal of endogenous cellular components to avoid inflammatory events and immune rejection [35]. Most published studies have used histological characterization or quantitative techniques to analyze the DNA removal [12,21]. Many decellularization protocols for hearts have been developed in recent years, with the most commonly applied technique being the Langendorff perfusion of decellularization agents via the ascending aorta [21,36].

The ultimate goal of regenerative medicine is to create bioartificial hearts generated from patients' cells. Several considerable barriers remain before this technology can be scaled up to human size and be of clinical relevance. Our work represents an important milestone in the quest for organ bioengineering using decellularized scaffolds in small-animal models. The decellularization protocol using an electric field developed by our research team can be modified for human-sized hearts. The Langendorff device prototype can be improved to provide the optimal flow rate for the human heart after obtaining the ethical approval for human tissue research. The results of the decellularization protocol based on the application of an electric field were comparable to those of current gold-standard decellularization techniques. The first experimental prototype of a tissue-engineered human-sized porcine whole-heart was conducted by Weymann et al. in 2014. Researchers used pigs to obtain an ECM scaffold because porcine cardiac anatomy is similar to that of humans [16]. Other mammal-derived organs have also been studied for future in vivo implantation in humans. Our improved methodology of decellularization can be used for other organs such as the kidney, liver or lung. Our results showed the 
optimization of the SDS exposure time using an alternating rectangular electric field on the preservation of ECM characteristics in whole-organ decellularization. Our results yielded an improved protocol (1\% SDS perfused for $8 \mathrm{~h}$ ), which uses a lower concentration of SDS than other works on whole-rat-heart decellularization, while being at least as effective in achieving the removal of cellular components.

Building a whole rat heart from decellularized scaffolds continues to be a challenge because it requires an enormous number of cells and most of them do not survive or differentiate into cardiomyocytes (CMs) during the recellularization process. The wholeheart engineering evolution started in 2008, when Ott et al. described the first rodent heart de- and recellularization process [10]. Recellularization of heart scaffolds was performed via direct injections into the ECM and infusion of endothelial cells via the coronary arteries [10,16,37]. In previous studies, cardiomyocytes differentiated from hMSCs [20], embryonic stem cells [19] and induced pluripotent stem cells (iPSCs) [21]. In the present study, hMSCs were used for seeding the decellularized ECM with the purpose of generating a bioartificial rodent heart and their pluripotency was confirmed using OM. 5-aza was used as a differentiation agent to induce hMSCs into CMs because it was shown to stimulate cardiomyogenesis [28,29]. Findings from our study should be widely applicable to decellularized whole-organ biological scaffolds in the development of regenerated organ replacements for transplantation.

Although an important fundamental parameter was examined (time exposure of the heart with SDS) within the decellularization process, there are many other important parameters, such as tissue harvesting factors, perfusion factors and sterilization methods, that inevitably affect the quality of the ECM scaffold. Another current limitation and a major area for future development is represented by the functional testing of the bioactivity of the ECM scaffold.

A future research direction would be a more comprehensive characterization of cardiomyocyte differentiation and electrical properties, as well as mechanical testing of the bioartificial heart.

\section{Conclusions}

In conclusion, the present study demonstrated an optimal and time-saving decellularization protocol using a modified Langendorff experimental device in the presence of an alternating rectangular electric field. For the recellularization of decellularized ECM, it was shown that hMSCs may be used to generate a bioartificial rat heart. However, major challenges remain in the construction of a functional tissue-engineered heart.

Author Contributions: Conceptualization, G.I.B., F.M.B., V.L.O. and O.I.G.; methodology, G.I.B., F.M.B. and V.P.; software, I.D.G., T.P.B., T.H. and V.L.O.; validation, F.M.B., O.I.G. and V.P.; formal analysis, G.I.B., I.D.G., T.P.B. and V.L.O.; investigation, G.I.B., F.M.B. and O.I.G.; resources, V.L.O., F.B., T.H. and V.P.; data curation, I.D.G., T.P.B. and V.L.O.; writing-original draft preparation, G.I.B., F.M.B., I.D.G. and F.B.; writing-review and editing, G.I.B., F.M.B. and V.P.; visualization, G.I.B., F.B. and F.M.B.; supervision, F.M.B. and V.P.; project administration, G.I.B., F.M.B., V.L.O. and T.H. All authors have read and agreed to the published version of the manuscript.

Funding: This research received no external funding.

Institutional Review Board Statement: The study was conducted according to the ARRIVE guidelines, and approved by the Institutional Ethics Committee of Clinical Emergency County Hospital "Pius Brinzeu" Timisoara within the project Development of BIOnanotechnologies based on Extracellular Vesicles, applied in early diagnostic, prognosis and therapy of atherosclerotic disease-BIOVEA (No. 162/01.07.2019) and by Institutional Ethics Committee of "Victor Babes" University of Medicine and Pharmacy Timisoara (No. 10/16.02.2021). The experiments were performed in an accredited facility and comply with the legal requirements on the laboratory animal study.

Informed Consent Statement: Not applicable.

Conflicts of Interest: The authors declare no conflict of interest. 


\section{References}

1. Cohn, J.N. Continue what we are doing to treat HF, but do it better. Nat. Rev. Cardiol. 2013, 11, 69-70. [CrossRef]

2. James, S.L.; Abate, D.; Abate, K.H.; Abay, S.M.; Abbafati, C.; Abbasi, N.; Abbastabar, H.; Abd-Allah, F.; Abdela, J.; Abdelalim, A.; et al. Global, regional, and national incidence, prevalence, and years lived with disability for 354 diseases and injuries for 195 countries and territories, 1990-2017: A systematic analy-sis for the Global Burden of Disease Study 2017. Lancet 2018, 392, 1789-1858. [CrossRef]

3. Tonsho, M.; Michel, S.; Ahmed, Z.; Alessandrini, A.; Madsen, J.C. Heart Transplantation: Challenges Facing the Field. Cold Spring Harb. Perspect. Med. 2014, 4, a015636. [CrossRef]

4. $\quad$ Rienks, M.; Papageorgiou, A.-P.; Frangogiannis, N.; Heymans, S. Myocardial Extracellular Matrix: An Ever-Changing and Diverse Entity. Circ. Res. 2014, 114, 872-888. [CrossRef] [PubMed]

5. Wang, B.; Tedder, M.E.; Perez, C.E.; Wang, G.; Curry, A.L.D.J.; To, F.; Elder, S.H.; Williams, L.N.; Simionescu, D.T.; Liao, J. Structural and biomechanical characterizations of porcine myocardial extracellular matrix. J. Mater. Sci. Mater. Med. 2012, 23, 1835-1847. [CrossRef] [PubMed]

6. Kaiser, N.J.; Coulombe, K.L.K. Physiologically inspired cardiac scaffolds for tailored in vivo function and heart regeneration. Biomed. Mater. 2015, 10, 034003. [CrossRef] [PubMed]

7. Taylor, D.A.; Sampaio, L.C.; Ferdous, Z.; Gobin, A.S.; Taite, L.J. Decellularized matrices in regenerative medicine. Acta Biomater. 2018, 74, 74-89. [CrossRef]

8. Chan, B.P.; Leong, K.W. Scaffolding in tissue engineering: General approaches and tissue-specific considerations. Eur. Spine J. 2008, 17, 467-479. [CrossRef] [PubMed]

9. Moser, P.T.; Ott, H.C. Recellularization of organs: What is the future for solid organ transplantation? Curr. Opin. Organ Transplant. 2014, 19, 603-609. [CrossRef]

10. Ott, H.C.; Matthiesen, T.S.; Goh, S.-K.; Black, L.D.; Kren, S.; Netoff, T.I.; Taylor, D.A. Perfusion-decellularized matrix: Using nature's platform to engineer a bioartificial heart. Nat. Med. 2008, 14, 213-221. [CrossRef]

11. Zia, S.; Mozafari, M.; Natasha, G.; Tan, A.; Cui, Z.; Seifalian, A. Hearts beating through decellularized scaffolds: Whole-organ engineering for cardiac regeneration and transplantation. Crit. Rev. Biotechnol. 2015, 36, 705-715. [CrossRef] [PubMed]

12. Sánchez, P.L.; Santos, M.E.F.; Costanza, S.; Climent, A.M.; Moscoso, I.; Gonzalez-Nicolas, M.A.; Sanz-Ruiz, R.; Rodríguez, H.; Kren, S.M.; Garrido, G.; et al. Acellular human heart matrix: A critical step toward whole heart grafts. Biomaterials 2015, 61, 279-289. [CrossRef] [PubMed]

13. Gilbert, T.; Sellaro, T.L.; Badylak, S.F. Decellularization of tissues and organs. Biomaterials 2006, 27, 3675-3683. [CrossRef]

14. Crapo, P.M.; Gilbert, T.; Badylak, S.F. An overview of tissue and whole organ decellularization processes. Biomaterials 2011, 32, 3233-3243. [CrossRef]

15. Weymann, A.; Loganathan, S.; Takahashi, H.; Schies, C.; Claus, B.; Hirschberg, K.; Soós, P.; Korkmaz, S.; Schmack, B.; Karck, M.; et al. Development and Evaluation of a Perfusion Decellularization Porcine Heart Model—Generation of 3-Dimensional Myocardial Neoscaffolds: Generation of 3-Dimensional Myocardial Neoscaffolds. Circ. J. 2011, 75, 852-860. [CrossRef]

16. Weymann, A.; Patil, N.P.; Sabashnikov, A.; Jungebluth, P.; Korkmaz, S.; Li, S.; Veres, G.; Soos, P.; Ishtok, R.; Chaimow, N.; et al. Bioartificial Heart: A Human-Sized Porcine Model-The Way Ahead. PLoS ONE 2014, 9, e111591. [CrossRef]

17. Park, S.M.; Yang, S.; Rye, S.-M.; Choi, S.W. Effect of pulsatile flow perfusion on decellularization. Biomed. Eng. Online 2018, 17, 15. [CrossRef] [PubMed]

18. Taylor, D.A.; Parikh, R.B.; Sampaio, L.C. Bioengineering Hearts: Simple yet Complex. Curr. Stem Cell Rep. 2017, 3, 35-44. [CrossRef]

19. Vats, A.; Tolley, N.S.; Bishop, A.E.; Polak, J.M. Embryonic stem cells and tissue engineering: Delivering stem cells to the clinic. J. R. Soc. Med. 2005, 98, 346-350. [CrossRef]

20. Toma, C.; Pittenger, M.F.; Cahill, K.S.; Byrne, B.J.; Kessler, P.D. Human Mesenchymal Stem Cells Differentiate to a Cardiomyocyte Phenotype in the Adult Murine Heart. Circulation 2002, 105, 93-98. [CrossRef]

21. Lu, T.-Y.; Lin, B.; Kim, J.; Sullivan, M.; Tobita, K.; Salama, G.; Yang, L. Repopulation of decellularized mouse heart with human induced pluripotent stem cell-derived cardiovascular progenitor cells. Nat. Commun. 2013, 4, 2307. [CrossRef]

22. Karantalis, V.; Hare, J.M. Use of Mesenchymal Stem Cells for Therapy of Cardiac Disease. Circ. Res. 2015, 116, 1413-1430. [CrossRef]

23. Ryan, J.M.; Barry, F.P.; Murphy, J.M.; Mahon, B.P. Mesenchymal stem cells avoid allogeneic rejection. J. Inflamm. $2005,2,8$. [CrossRef] [PubMed]

24. Hahn, J.-Y.; Cho, H.-J.; Kang, H.-J.; Kim, T.-S.; Kim, M.-H.; Chung, J.-H.; Bae, J.-W.; Oh, B.-H.; Park, Y.-B.; Kim, H.-S. Pre-Treatment of Mesenchymal Stem Cells With a Combination of Growth Factors Enhances Gap Junction Formation, Cytoprotective Effect on Cardiomyocytes, and Therapeutic Efficacy for Myocardial Infarction. J. Am. Coll. Cardiol. 2008, 51, 933-943. [CrossRef] [PubMed]

25. Gu, L.-H.; Zhang, T.-T.; Li, Y.; Yan, H.-J.; Qi, H.; Li, F.-R. Immunogenicity of allogeneic mesenchymal stem cells transplanted via different routes in diabetic rats. Cell. Mol. Immunol. 2014, 12, 444-455. [CrossRef]

26. Bonciog, D.D.; Matiu-Iovan, L.; Barbulescu, G.-I.; Burian, C.A.; Goje, I.-D.; Buica, T.P.; Paunescu, V.; Ordodi, V.L. Modified Langendorff Device for Rat Heart Decellularization. Physiology 2019, 2, 17-20. 
27. Bonciog, D.D.; Lascu, M.R.; Matiu-Iovan, L.; Ionel, R.; Burian, C.A.; Ordodi, V.L. Modified Langendorff vibrating fluid column device for improved rat heart decellularization experiments. In Proceedings of the 2020 International Symposium on Electronics and Telecommunications (ISETC), Timisoara, Romania, 5-6 November 2020.

28. Xu, W.; Zhang, X.; Qian, H.; Zhu, W.; Sun, X.; Hu, J.; Zhou, H.; Chen, Y. Mesenchymal Stern Cells from Adult Human Bone Marrow Differentiate into a Cardiomyocyte Phenotype In Vitro. Exp. Biol. Med. 2004, 229, 623-631. [CrossRef]

29. Antonitsis, P.; Ioannidou-Papagiannaki, E.; Kaidoglou, A.; Papakonstantinou, C. In vitro cardiomyogenic differentiation of adult human bone marrow mesenchymal stem cells. The role of 5-azacytidine. Interact. Cardiovasc. Thorac. Surg. 2007, 6, 593-597. [CrossRef] [PubMed]

30. Iop, L.; Sasso, E.D.; Menabò, R.; Di Lisa, F.; Gerosa, G. The Rapidly Evolving Concept of Whole Heart Engineering. Stem Cells Int. 2017, 2017, 8920940. [CrossRef]

31. Lunkenheimer, P.P.; Redmann, K.; Westermann, P.; Rothaus, K.; Cryer, C.W.; Niederer, P.; Anderson, R.H. The myocardium and its fibrous matrix working in concert as a spatially netted mesh: A critical review of the purported tertiary structure of the ventricular mass. Eur. J. Cardio-Thoracic Surg. 2006, 29, S41-S49. [CrossRef]

32. Costa, K.D.; Lee, E.J.; Holmes, J. Creating Alignment and Anisotropy in Engineered Heart Tissue: Role of Boundary Conditions in a Model Three-Dimensional Culture System. Tissue Eng. 2003, 9, 567-577. [CrossRef]

33. Eschenhagen, T.; Zimmermann, W.H. Engineering Myocardial Tissue. Circ. Res. 2005, 97, 1220-1231. [CrossRef] [PubMed]

34. Ye, X.; Wang, H.; Gong, W.; Li, S.; Li, H.; Wang, Z.; Zhao, Q. Impact of decellularization on porcine myocardium as scaffold for tissue engineered heart tissue. J. Mater. Sci. Mater. Electron. 2016, 27, 70. [CrossRef] [PubMed]

35. Badylak, S.F.; Gilbert, T. Immune response to biologic scaffold materials. Semin. Immunol. 2008, 20, 109-116. [CrossRef]

36. Remlinger, N.T.; Wearden, P.D.; Gilbert, T.W. Procedure for Decellularization of Porcine Heart by Retrograde Coronary Perfusion. J. Vis. Exp. 2012, e50059. [CrossRef]

37. Robertson, M.J.; Dries-Devlin, J.L.; Kren, S.M.; Burchfield, J.S.; Taylor, D.A. Optimizing Recellularization of Whole Decellularized Heart Extracellular Matrix. PLoS ONE 2014, 9, e90406. [CrossRef] [PubMed] 